\title{
Research Article \\ Experimental Study on Thermal Performance of a Bent Copper-Water Heat Pipe
}

\author{
Shuangshuang Miao, ${ }^{1}$ Jiajia Sui, ${ }^{1}$ Yulong Zhang, ${ }^{2}$ Feng Yao $\mathbb{D}^{1,3}$ and Xiangdong Liu $\mathbb{D}^{1,4}$ \\ ${ }^{1}$ Key Laboratory of Energy Thermal Conversion and Control of Ministry of Education, School of Energy and Environment, \\ Southeast University, Nanjing, Jiangsu 210096, China \\ ${ }^{2}$ Pen-Tung Sah Institute of Micro-Nano Science and Technology, Xiamen University, Xiamen 361005, China \\ ${ }^{3}$ Jiangsu Key Laboratory of Micro and Nano Heat Fluid Flow Technology and Energy Application, School of Environmental Science \\ and Engineering, Suzhou University of Science and Technology, Suzhou, Jiangsu 215009, China \\ ${ }^{4}$ College of Electrical, Energy and Power Engineering, Yangzhou University, Yangzhou 225127, China
}

Correspondence should be addressed to Feng Yao; yaofeng@usts.edu.cn and Xiangdong Liu; liuxd@yzu.edu.cn

Received 1 January 2020; Accepted 28 April 2020; Published 30 June 2020

Academic Editor: Chengbin Zhang

Copyright (c) 2020 Shuangshuang Miao et al. This is an open access article distributed under the Creative Commons Attribution License, which permits unrestricted use, distribution, and reproduction in any medium, provided the original work is properly cited.

\begin{abstract}
Vapor-liquid phase change is regarded as an efficient cooling method for high-heat-flux electronic components. The copper-water bent heat pipes are particularly suited to the circumstances of confined space or misplaced heat and cold sources for high-heat-flux electronic components. In this paper, the steady and transient thermal performance of a bent copper-water heat pipe is studied based on a performance test system. The effects of cooling temperature, working conditions on the critical heat flux, and equivalent thermal conductivity have been examined and analyzed. Moreover, the influences of heat input and working conditions on the thermal response of a bent heat pipe have also been discussed. The results indicate that the critical heat flux is enhanced due to the increases in cooling temperature and the lengths of the evaporator and condenser. In addition, the critical heat flux is improved by extending the cooling length only when the operating temperature is higher than $50^{\circ} \mathrm{C}$. The improvement on the equivalent thermal by increasing the heating length is more evident than that by increasing cooling length. It is also demonstrated by the experiment that the bent copper-water heat pipe can respond quickly to the variation of heat input and possesses superior transient heat transfer performance.
\end{abstract}

\section{Introduction}

In the last decade, with the rapid development of microelectronic technology, there is an irreversible development trend towards to the high integration, miniaturization, and high power for the electronic components [1-4]. Vapor-liquid phase change is regarded as an efficient cooling method for high-heat-flux electronic components [5-7], because excessive temperature leads to the unstable working status and shortens the life of electronic components, resulting in a decrease in system reliability $[8,9]$. For example, if the operating temperature increases by $10^{\circ} \mathrm{C}$, the reliability of electronic components will be reduced by $50 \%$ [10]. Therefore, in order to avoid this unfavorable fact, several advanced high-heat-flux electronic component cooling technologies have been developed recently, including direct immersion boiling, jet, and heat pipe [11-14]. Among them, the heat pipe has already been widely applied in thermal control systems owing to high thermal conductivity and superior isothermal properties. And it has been a hotpot in the research area of heat transfer enhancement.

Nowadays, compared with traditional cooling methods such as air cooling, the straight heat pipes have been one of the common and important options for heat dissipation technologies attributed to the advantages of high transmission, compact structure, and small volume-to-mass ratio [15-18]. However, the utilization of a straight heat pipe is restricted in certain circumstances such as the confined space 
for heat pipe installation or the occasion that the heat source and the cold source are misplaced. In some specific situations, it is necessary to bend the heat pipe into a $U$ shape to match the application requirements. Fortunately, bending the heat pipe may also have some positive effects, such as the heat transfer can be enhanced by taking advantage of the gravitational field. Jankowski et al. [19] tried to apply the bent heat pipes to rotating machinery such as motors and engines, and they built a rotating test platform in which both the evaporator section and the condenser area are paralleled to the axis. The results indicate that the working medium in the bent heat pipe can keep circulating, and the thermal performance of the bent heat pipe keeps reliable even in a rotating state. Yang and Chiang [20] studied the heat transfer characteristics and fluid flow friction of a bent heat pipe with wave shape, and the impact of the curvature radius on the average heat transfer coefficient and friction coefficient was studied. It is observed that the friction coefficient of the curved heat pipe is increased by nearly $40 \%$, and the heat transfer rate can be enhanced by $100 \%$. Therefore, it is promising that the performance of the heat exchanges or the solar collector can be enhanced by replacing the straight pipe with the S-type bent heat pipe.

Inside a heat pipe, the circulation of gas-liquid two-phase flow is dominated by the wick-generated capillary force. So, the wick is one of the significant properties for a heat pipe. The surface capillary $r_{C}$ and permeability $K$ are the two main characteristic parameters of the capillary wick [21-23]. $r_{C}$ determines the maximum capillary pressure $P_{C, \max }$ of the capillary wick, which characterizes the driving force for the circulation of the wick heat pipe, and $K$ determines the frictional resistance loss of condensate in the wick, which characterizes the heat pipe flow resistance. The wick is important because it serves two purposes for the heat pipe. One purpose is providing paths for the backflow of the condensate from condensation area to evaporation area and the paths for heat conduction from the wall to the gas-liquid interface. The other purpose is providing surface capillary pores that can generate capillary pumping force for fluid flow [24]. Based on these demands, the screen mesh, glass fiber, sintered porous metal, and microchannels are chosen as the main materials of the wick [25]. However, not all types of the wick above can be applied to the bent heat pipe. For example, the bending of the screen mesh easily breaks the metal mesh structure and deteriorates the heat transfer performance, and similarly, a structure damage problem appears when bending other types of wick such as glass fiber, microchannel and flat-plate heat pipe [26]. Yu et al. [27] studied the evaporation regime of the microchannel heat pipe at small tilt angles and found that bending the heat pipe would change the evaporation regime. On the contrary, when applied to the bent heat pipe, the metal sintered powder exhibits an unprecedented advantage over the other types of wick. Moreover, the thermal performance of the heat pipe with sintered powder wick hardly decreases even after multiple bending. As a result, due to its high thermal conductivity and high capillary pumping force, the metal sintered powder wick has been recognized as an ideal choice for the wick of the bent heat pipe.
Nowadays, more attention has been paid from the straight heat pipes toward the bent heat pipes owing to the complex application environment. However, due to the special-shaped structure of the bent heat pipe, the vaporliquid two-phase fluid flow inside the bent heat pipe is complicated, resulting in difficulty in understanding thermal performance through theoretical investigation. In recent years, there have been some researches on bent heat pipes. Wang [28] focused on the transient response characteristics of the bent copper-water heat pipe with a grooved inner surface, while Yang et al. [29] studied the effect of a bent angle on heat transfer performance by bending the adiabatic section of the copper mesh heat pipe, which shows that their bent heat pipes have less thermal resistance and better flexibility than polymer heat pipes. Recently, the effect of bending angles on the heat transfer performance of the heat pipe has been studied; the researches show that increasing bending angles will increase the heat transfer resistance of the heat pipe [30]. In addition, Jiang et al. [31] found that the heat transfer capacity of the heat pipe decreases when the bending position is closer to the evaporator section. Although there have been some researches on bent heat pipes, but relatively few, the current understanding of the heat transfer performance of bent heat pipes is still insufficient. Therefore, in-depth researches still need to be conducted to comprehensively understand the thermal performance of a bent heat pipe by using an experimental test. In view of this, an experimental system focused on the thermal performance of a bent copper-water heat pipe has been built in this paper. The critical heat flux, operating temperature, and equivalent thermal conductivity of a bent copper-water heat pipe under steady state have been experimentally studied and analyzed. According to the existing literature [32], an extension of evaporation and condensation processes into the adiabatic section due to the heat conduction of the heat pipe wall can be found. In other words, the heat transfer and fluid flow near the joint of one section and another (e.g., the evaporator section and adiabatic section) will be affected by changing the length of the evaporator section $\left(\mathrm{L}_{\mathrm{e}}\right)$ and the length of the condenser section $\left(\mathrm{L}_{\mathrm{c}}\right)$. In particular, when the bending position is near the joint, the local heat transfer and fluid flow will surely be affected by the bending structure. Therefore, the study on the effect of $\mathrm{L}_{\mathrm{e}}$ and $\mathrm{L}_{\mathrm{c}}$ on the heat transfer performance is important for the application of the bent heat pipe, which will be conducted in this paper. Furthermore, the transient temperature profiles of the bent heat pipe have also been obtained and discussed. This study is motivated to provide basic experimental study data for the application of bent heat pipes.

\section{Experimental Setup}

As shown in Figure 1, the experimental system includes a bent copper-water heat pipe, an electric heating lock, a DC power supply, a power meter, a constant temperature water bath, a data acquisition instrument, a computer, and several thermocouples. The electric heating block is composed of two electric heating rods and an aluminum substrate. The experimental system rig is shown in Figure 1(b). However, 


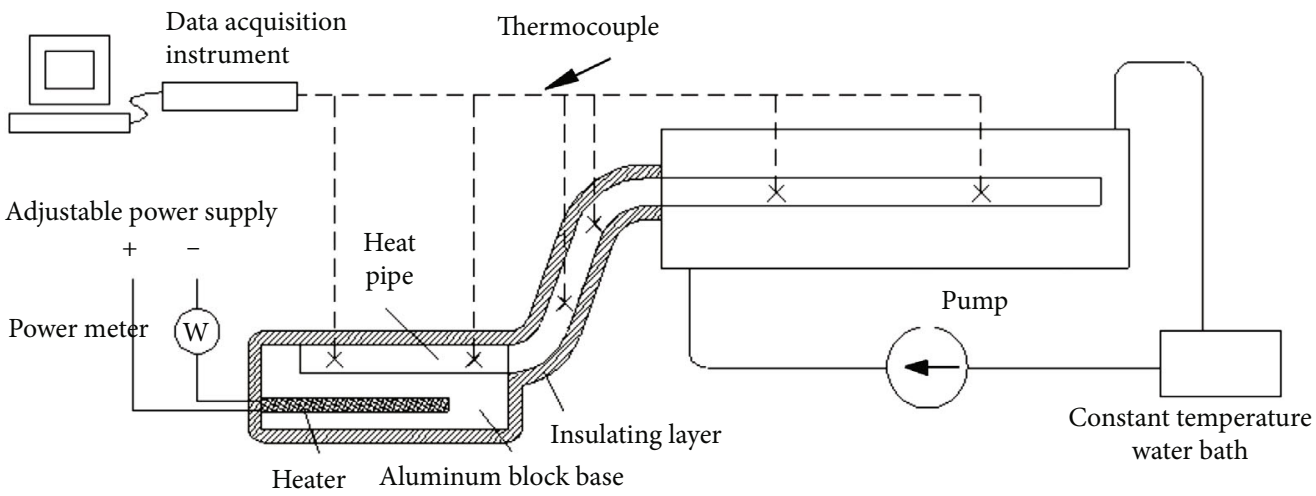

(a)



(b)

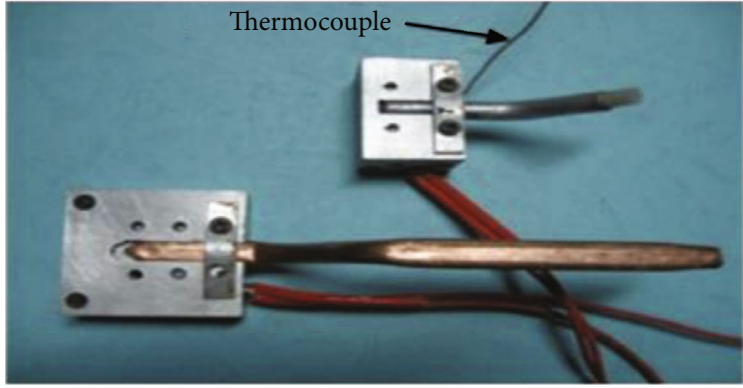

(d)

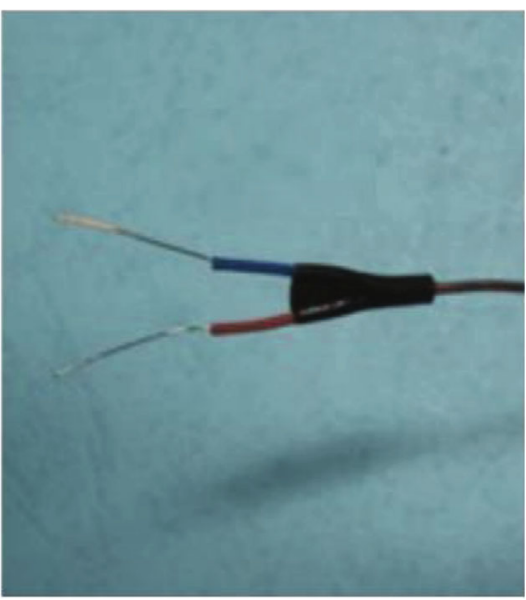

(c)

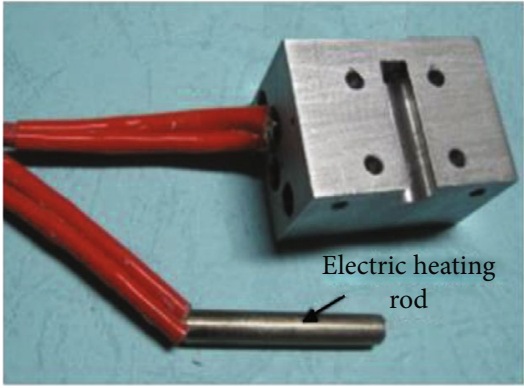

(e)

FIGURE 1: Experimental system of the bent heat pipe: (a) experimental schematic, (b) experimental system rig, (c) thermocouples, (d) metal sintered powder heat pipe, and (e) electric heating rod.

as it is difficult to get clear insight into the test section from Figure 1(b), the detailed structure of the test section including thermal couples, heat pipe, heating block, and electric heating rod has been shown in Figures $1(\mathrm{c})-1(\mathrm{e})$ additionally. It should be pointed out that the isolation layer, which wrapped the heating section of the heat pipe during the experiment, was removed when taking pictures for better observation.

The high heat flux is realized by the heating block which is inserted with several heating rods. And the constant temperature water bath provides the cooling water as the cold source. During the experiment, the operating condition of the bent heat pipe is changed by adjusting the heating power of the electric heating block and the water temperature. The heating power is measured by the power meter. The temperature distribution is measured by the thermocouples that are axially fixed on the solid surface of the heat pipe. On each section, i.e., evaporator section, adiabatic section, and condenser section, 2 thermocouples are arranged. And the operating temperature is defined as the arithmetic mean value of the tested wall temperature.

The actual transmission power of the copper-water bent heat pipe is obtained by subtracting the heat loss induced by the natural convection on the surface from the heating power. The characteristic correlation of the natural convective heat transfer coefficient of constant wall temperature in large space is 


$$
\mathrm{Nu}_{m}=c(\mathrm{GrPr})_{m}^{n}
$$

Therefore, the amount of heat loss caused by the natural convection heat transfer outside the bent heat pipe insulation layer is

$$
Q_{\text {loss }}=h A_{\text {out }} \Delta t=\mathrm{Nu}_{m} \frac{K_{\mathrm{f}}}{l_{\mathrm{c}}} A_{\text {out }} \Delta t
$$

where $\mathrm{Gr}$ is the Grashof number, $\mathrm{Gr}=g \alpha_{\mathrm{v}} \Delta t l_{\mathrm{c}}{ }^{3} / \gamma^{2}$. Pr is the Prandtl number, $\operatorname{Pr}=\gamma / a$. $\mathrm{Nu}$ is the Nusself number, $\mathrm{Nu}=$ $h l_{\mathrm{c}} / K_{\mathrm{f}} . \alpha_{\mathrm{v}}$ is the volume expansion coefficient of the gas, for ideal gas $\alpha_{\mathrm{v}}=1 / T_{m}\left(\mathrm{~K}^{-1}\right) . \Delta t$ is the temperature difference of convective heat transfer. In this experiment, the fluid is heated, $\Delta t=t_{\mathrm{w}}-t_{\infty}$, where $t_{\mathrm{w}}$ is the temperature of the outer surface of the insulation layer of the bent heat pipe evaporation section and the adiabatic section and $t_{\infty}$ is the distance away from the wall fluid temperature. $l_{c}$ is the characteristic size; after the insulation layer is wrapped, the shape of the evaporation section and adiabatic section of the bent heat pipe is approximately a rectangular parallelepiped, so the equivalent diameter $d$ of the rectangular is taken as $l_{c}$. $A_{\text {out }}$ is the outer surface area of the evaporation section and adiabatic section of the bent heat pipe after being wrapped with an insulation layer $\left(\mathrm{m}^{2}\right) . g$ is the gravitational acceleration, taken as $9.807 \mathrm{~m} / \mathrm{s}^{2} . a$ is the thermal diffusivity $\left(\mathrm{m}^{2} / \mathrm{s}\right) \cdot \gamma$ is the kinematic viscosity $\left(\mathrm{m}^{2} / \mathrm{s}\right) . K_{\mathrm{f}}$ is the thermal conductivity of the fluid $(\mathrm{W} /(\mathrm{m} \cdot \mathrm{K})), 0.0267 \mathrm{~W} /\left(\mathrm{m}^{2} \cdot \mathrm{K}\right)$ at $20^{\circ} \mathrm{C}$ air. $h$ is the convective heat transfer coefficient $\left(\mathrm{W} /\left(\mathrm{m}^{2} \cdot \mathrm{K}\right)\right) . c$ and $n$ are the coefficient and indice determined by experiments. In this experiment, the values are $c=0.48, n=0.25,10^{4} \leq \mathrm{GrPr} \leq$ $1.5 * 10^{8}$, laminar flow. $T_{m}$ is the characteristic temperature, $T_{m}=\left(t_{\mathrm{w}}+t_{\infty}\right) / 2(\mathrm{~K})$.

The uncertainty on the thermodynamic properties of air is determined based on recommendations in open literature $[33,34]$. For example, in the case of $t_{\mathrm{w}}=60^{\circ} \mathrm{C}$, the heat loss $Q_{\text {loss }}$ calculated according to the above formula is $0.10518 \mathrm{~W}$.

Standard error propagation rules as described by Moffat [35] were used to calculate the overall uncertainty, which is the root-sum-square method. In this experiment, all thermocouples were calibrated to systematic errors before measurements. Taking into consideration the contact thermal resistance, the resulting uncertainty on all used temperatures varies within $0.75^{\circ} \mathrm{C}$. The uncertainty of heat flux is within $3 \%$. The uncertainty of the equivalent thermal conductivity is within $5 \%$.

In this experiment, aiming at the impact of evaporator length and condenser length on the thermal performance of a bent copper-water heat pipe, various combinations of different evaporator and condenser lengths have been adopted. The distribution of the evaporator area and the condenser area (directly reflected by the length) is shown in Table 1 .

Figure 2 illustrates the bent heat pipe for testing in the experiment. The bent heat pipe utilizing copper powder sintered wick is made of copper. The water is utilized as the working medium inside the heat pipe. Aiming at fully understanding the importance of working conditions that affect the
TABLE 1: Various combinations of different evaporator and condenser lengths.

\begin{tabular}{lcc}
\hline $\begin{array}{l}\text { Working } \\
\text { condition }\end{array}$ & $\begin{array}{c}\text { Evaporation } \\
\text { section }(\mathrm{cm})\end{array}$ & $\begin{array}{c}\text { Condensation } \\
\text { section }(\mathrm{cm})\end{array}$ \\
\hline$\# 1$ & 3 & 7 \\
$\# 2$ & 2 & 7 \\
$\# 3$ & 2 & 3.5 \\
$\# 4$ & 1 & 3.5 \\
\hline
\end{tabular}

thermal performance of the bent heat pipe, the effects of the lengths of the evaporator and condenser, the cooling temperature on the effective heat conductivity, and critical heat flux have been investigated; the temperature response of the bent heat pipe under high heating power has also been studied.

\section{Results and Discussion}

The reliable applicability of the heat pipe under high-heatflux conditions depends on the heat transfer limit, i.e., the critical heat flux. Secondly, the temperature uniformity is characterized by the equivalent thermal conductivity. In addition, the temperature response shows the transient heat transfer characteristics of the bent copper-water heat pipe. Therefore, the above three characteristic parameters are introduced as the evaluation criterions of the bent heat pipe thermal performance.

\subsection{Steady-State Performance}

3.1.1. Heat Transfer Capacity and Cooling Temperature. When the heat input of the bent heat pipe reaches a specific threshold value, a small amount heat input increment $\Delta Q$ will cause a sudden rise in the wall temperature at the hot end of the evaporator section, which means that the evaporation section is partially or completely dry out attributing to the absence of backflow liquid. At this time, the heat transfer limit, i.e., the critical heat flux $q_{\mathrm{cr}}$, is considered to be reached. Once the heat flux is larger than the $q_{\mathrm{cr}}$, the performance of the bent heat pipe will rapidly deteriorate and cannot work stably anymore. Therefore, $q_{\mathrm{cr}}$ is important for the design of the bent heat pipe. The heat pipe operating temperature $t$ is defined as the average value of the temperature at evaporation section $t_{\mathrm{e}}$, temperature at adiabatic section $t_{\mathrm{a}}$, and temperature at condensing section $t_{\mathrm{c}}$. Operating temperature is also crucial to the application of the bent copper-water heat pipe.

Obviously, the heat transfer capability is not only related to the temperature of the cold source but also related to the area of the evaporation section that receives the energy and the area of the condensation section that releases the energy. Therefore, the effect of cooling temperature on $q_{\mathrm{cr}}$ and working temperature of the bent heat pipe with different evaporator and condenser lengths has been tested, as illustrated in Figure 3. As illustrated, the critical heat flux is greatly affected by the temperature of the cold source. $q_{\mathrm{cr}}$ has an approximately linear growth as the cooling temperature increases, indicating that higher operating temperature 


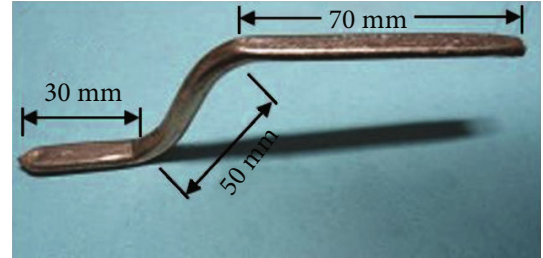

(a)

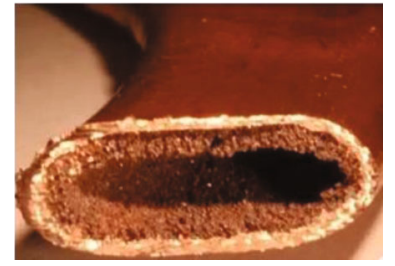

(b)

Figure 2: Heat pipe: (a) bent copper-water heat pipe; (b) the metal sintered powder wick.


(a)
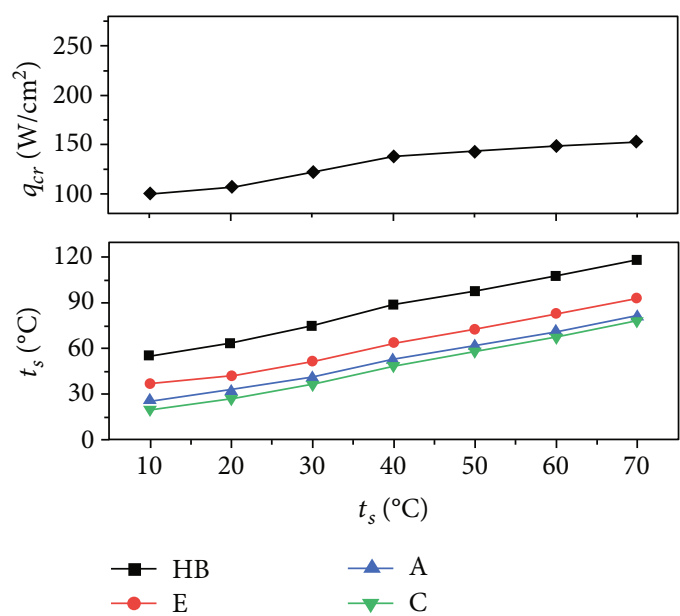

(c)
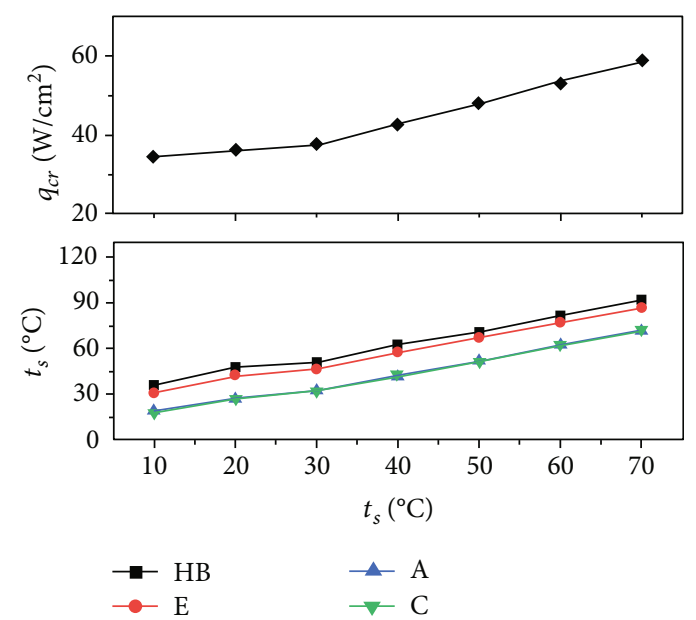

(d)

FIGURE 3: The critical heat flux and working temperature of a bent copper-water heat pipe with different evaporator and condenser lengths: (a) $L_{\mathrm{e}}=3 \mathrm{~cm}, L_{\mathrm{c}}=7 \mathrm{~cm}$, (b) $L_{\mathrm{e}}=2 \mathrm{~cm}, L_{\mathrm{c}}=7 \mathrm{~cm}$, (c) $L_{\mathrm{e}}=2 \mathrm{~cm}, L_{\mathrm{c}}=3.5 \mathrm{~cm}$, and (d) $L_{\mathrm{e}}=1 \mathrm{~cm}, L_{\mathrm{c}}=3.5 \mathrm{~cm}$. HB: heating block; E: evaporation; A: adiabatic; C: condensation.

(which increases with the rise of cooling temperature) is favorite to achieve the higher heat transfer limit due to more intense gas-liquid phase change processes inside the bent heat pipe. On the contrary, the critical heat flux is weakened under low-temperature conditions. On the other hand, the average temperature of the evaporation area, adiabatic area, and condensation area rises correspondingly when the cooling temperature is becoming higher, indicating that the temperature difference between condenser and evaporator has little relation to cooling temperature and remains almost unchanged. However, obvious distinctions of the critical heat flux can be observed among the bent heat pipes with different $L_{\mathrm{e}}$ and $L_{\mathrm{c}}$, which will be discussed in the following section.

More clear comparison of the critical heat flux of the bent copper-water heat pipes with various heating and cooling lengths is illustrated in Figure 4. From the comparative study, it is apparent that the lengths of the evaporator area and condenser area play an essential role on the heat pipe critical heat 


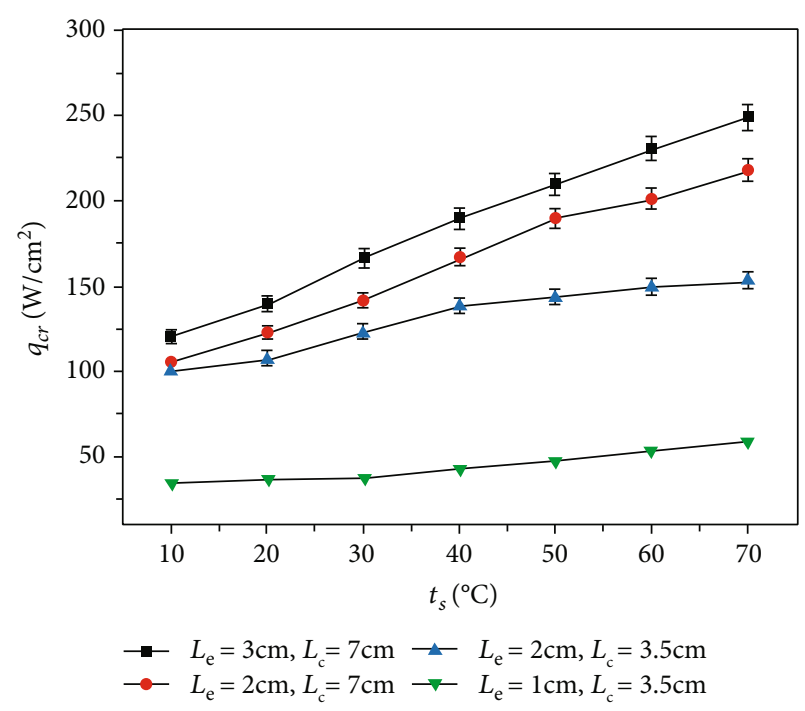

FIGURE 4: Effect of cooling temperature on the critical heat flux.

flux. As seen, the heat transfer capability of the original one $\left(L_{\mathrm{e}}=1 \mathrm{~cm}, L_{\mathrm{c}}=3.5 \mathrm{~cm}\right)$ is less than $30 \mathrm{~W}$ even though the cooling temperature has been raised to $70^{\circ} \mathrm{C}$. And $q_{\mathrm{cr}}$ can be undoubtedly improved by increasing both lengths of evaporator and condenser sections. However, it should be noted that, when the other conditions remain unchanged, $q_{\mathrm{cr}}$ can be significantly enhanced when the evaporator section length is doubled regardless of changing the cooling temperature. In contrast to the above phenomenon, only when the cooling temperature is about $50^{\circ} \mathrm{C}$ or higher, there will be a considerable improvement of $q_{\mathrm{cr}}$ by doubling the area of the condenser section. In other words, the effect of improving $q_{\mathrm{cr}}$ by increasing the cooling area is limited when the working temperature is low. This phenomenon can be explained by the fact that more heat input is required to reach the critical heat flux that the "dry out" phenomenon occurs by the increase of the evaporator area with the same condenser area; therefore, the heat transfer limit can be improved. If the heating area remains the same and the condenser area increases, more liquid can be formed by the condensation of vapor, which means that if the capillary pressure increases and the mass flow rate of condensate will be increased, more liquid can flow back to the evaporator for the supplement of evaporation; hence, the capillary limit is lifted. Furthermore, when the working temperature is becoming higher, both evaporation and condensation are intensified and the boiling limit $Q_{b, \max }$, capillary limit $Q_{c, \max }$, and entrainment limit $Q_{e, \max }$ are lifted, leading to a rather high heat transport ability. As a result, the critical heat flux under high working temperature obviously increases for the bent heat pipe with a longer length of the condenser section.

Figure 5 shows the operating temperature as a function of the cold source temperature for the bent copper-water heat pipes with different evaporator and condenser lengths. As shown in Figure 5, there is a good linear relationship between cooling temperature and operating temperature. In addition, the operating temperature is approximately equal to the tem-

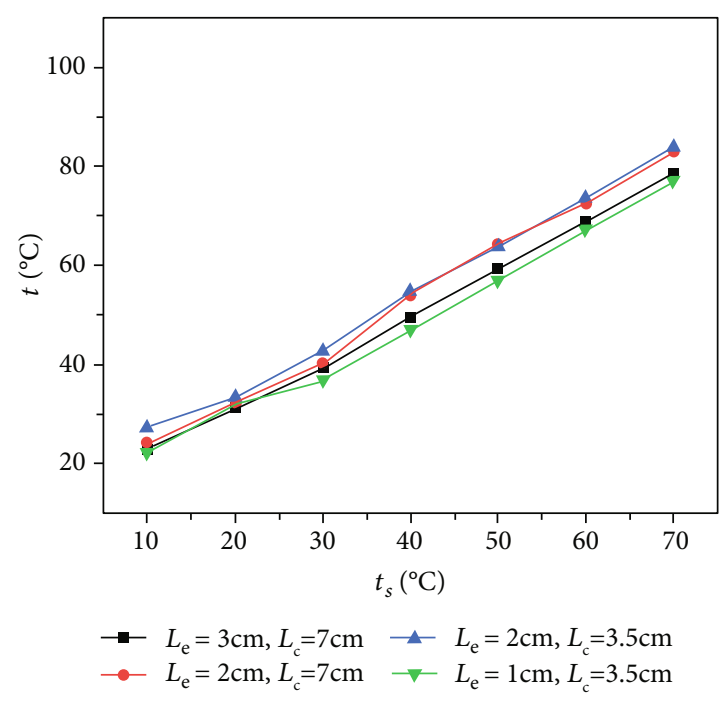

FIGURE 5: Effect of cooling temperature on operating temperature.

perature of the cold source, due to the superior isothermal characteristics of the bent copper-water heat pipe.

3.1.2. Equivalent Thermal Conductivity. The thermal performance of the bent copper-water heat pipe relies on the combined effects of the heat conduction and convection heat transfer between the metal shell and internal medium, inspired by the calculation method of the equivalent thermal conductivity of the porous medium and the concept of thermal resistance. For convenience, the total heat flux transferred is still calculated according to Fourier's law, which is $Q_{\text {in }}=\left(t_{\mathrm{w}, \mathrm{e}}-t_{\mathrm{w}, \mathrm{c}}\right) / R$ and $R=L_{\text {eff }} / k_{\text {eff }} A$. Therefore, the equivalent thermal conductivity is

$$
k_{\text {eff }}=\frac{Q_{\text {in }} L_{\text {eff }}}{A\left(t_{\mathrm{w}, \mathrm{e}}-t_{\mathrm{w}, \mathrm{c}}\right)}=\frac{Q_{\mathrm{in}} L_{\mathrm{eff}}}{A t_{\mathrm{e}-\mathrm{c}}},
$$

where $A$ is the cross-sectional area, $Q_{\text {in }}$ is the input power of the bent heat pipe, and the effective length is $L_{\text {eff }}=\left(L_{\mathrm{e}}+L_{\mathrm{c}}\right) / 2+L_{\mathrm{a}} \cdot t_{\mathrm{w}, \mathrm{e}}$ and $t_{\mathrm{w}, \mathrm{c}}$ are the mean temperature of the evaporator and condenser sections, respectively. Besides, $L_{\mathrm{e}}, L_{\mathrm{a}}$, and $L_{\mathrm{c}}$ are the lengths of the evaporation, adiabatic, and condenser sections, respectively.

Figure 6(a) describes the effect of cold source temperature on the equivalent thermal conductivity of the bent heat pipes with different $L_{\mathrm{e}}$ and $L_{\mathrm{c}}$. With the rise of cold source temperature, the equivalent thermal conductivity increases. In addition, the lengths of the evaporator and condenser sections show a more significant influence on the equivalent thermal conductivity. However, the extension of the evaporator section shows a more noticeable effect on the thermal conductivity, as compared with the condenser section. For example, the equivalent thermal conductivity will be about 1.4 times larger when the evaporator length is extended by 1.5 times (from $2 \mathrm{~cm}$ to $3 \mathrm{~cm}$ ). Yet, the equivalent thermal conductivity only shows a 1.2 times increment though the condenser length has been doubled 

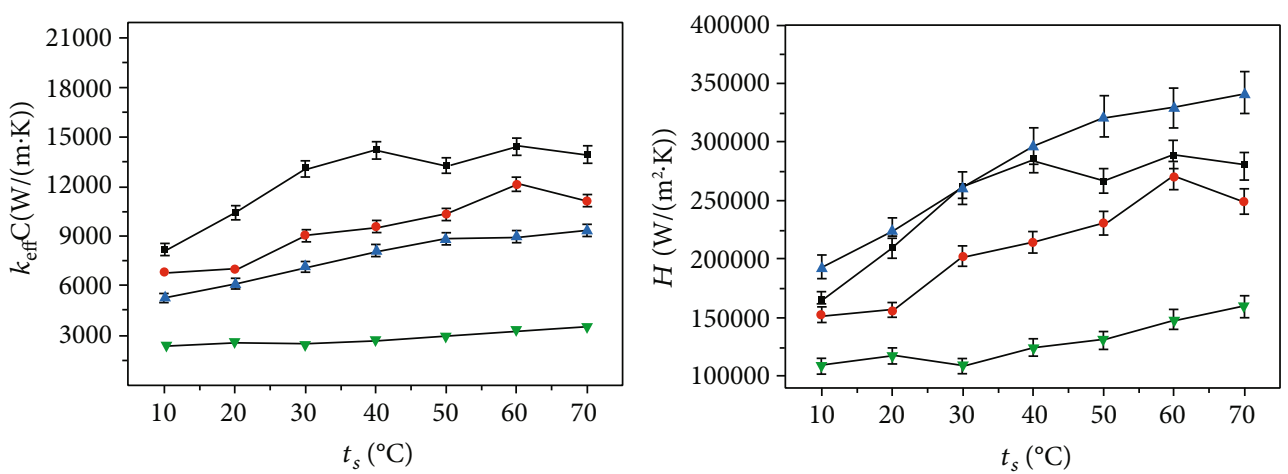

$\rightarrow L_{e}=3 \mathrm{~cm}, L_{c}=7 \mathrm{~cm} \rightarrow L_{e}=2 \mathrm{~cm}, L_{c}=3.5 \mathrm{~cm}$
$\rightarrow-L_{e}=2 \mathrm{~cm}, L_{c}=7 \mathrm{~cm} \rightarrow L_{e}=1 \mathrm{~cm}, L_{c}=3.5 \mathrm{~cm}$

Figure 6: Effect of cooling temperature on equivalent thermal conductivity (a) and performance-price coefficient (b) with different evaporator and condenser lengths.

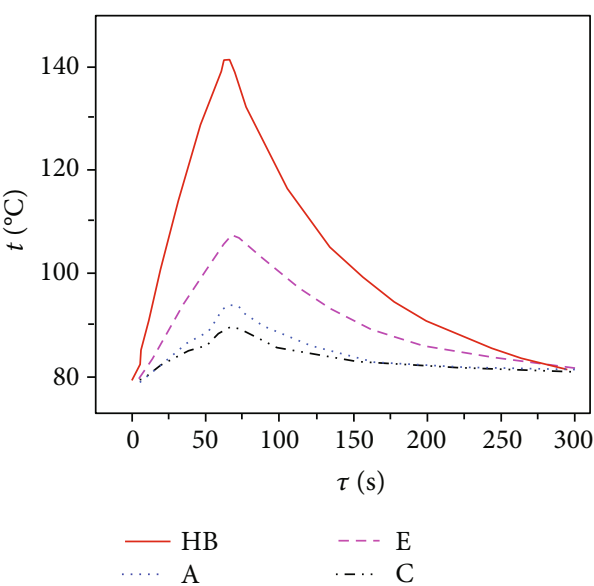

(a)

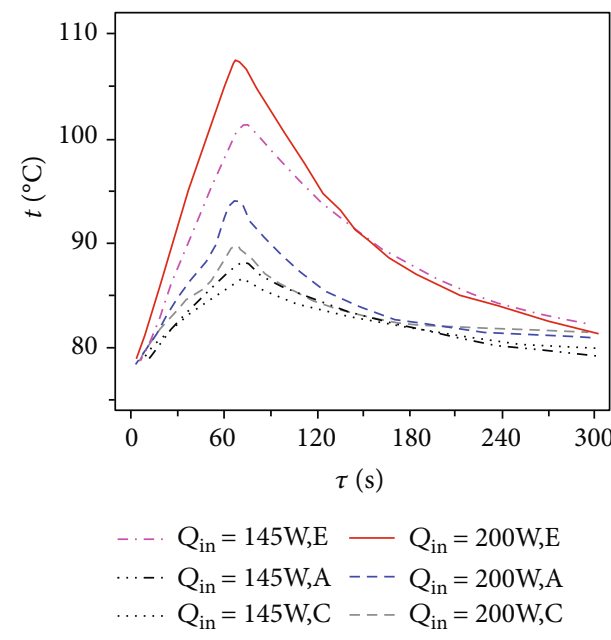

(b)

Figure 7: Behavior of thermal response: (a) transient temperature profiles of different parts of the bent heat pipe (HB: heating block; E: evaporation; A: adiabatic; C: condensation) and (b) effect of heat input on the thermal response of the bent heat pipe.

(from $3.5 \mathrm{~cm}$ to $7 \mathrm{~cm}$ ). Therefore, it can be concluded that rather than increasing the cooling length, it is a better choice to ensure that the heating length is as long as possible for heat transfer enhancement when focusing on the equivalent thermal conductivity. Inspired by the experiments, comprehensively considering the economic efficiency and heat transfer capacity, we give an index to judge the comprehensive performance of the bent heat pipe, which is a performance-price coefficient:

$$
H=\frac{k_{\text {eff }}}{\left(c_{1} L_{\mathrm{e}}+c_{2} L_{\mathrm{c}}\right)},
$$

where $c_{1}, c_{2}$ are relative to the relative prices of the materials selected for evaporator and condenser sections, $c_{1+} c_{2}=1$. In our experiment, $c_{1}=c_{2}=1 / 2$. The unit of the performanceprice coefficient is $\mathrm{W} /\left(\mathrm{m}^{2} \cdot \mathrm{K}\right)$. It can be seen from the formula that the less material used and the higher equivalent thermal conductivity lead to the larger performance-price coefficient. In our paper, the coefficient of the bent heat pipe with $L_{\mathrm{e}}=2 \mathrm{~cm}, L_{\mathrm{c}}=3.5 \mathrm{~cm}$ is the highest and should be selected among these four types, as shown in Figure 6(b). In this test, the equivalent thermal conductivity of the bent heat pipe is about $2000 \sim 18000 \mathrm{~W} /(\mathrm{m} \cdot \mathrm{K})$, which is about 100 times larger than the thermal conductivity of pure copper $(386.4 \mathrm{~W} /(\mathrm{m} \cdot \mathrm{K}))$. Consequently, the bent heat pipe exhibits superior thermal conductivity and temperature uniformity.

3.2. Thermal Response Behaviors. The transient characteristics are also crucial for the design and application of the bent copper-water heat pipe. Figure 7(a) illustrates the transient temperature profiles of the heating block $(\mathrm{HB})$, the evaporation section ( $\mathrm{E}, L_{\mathrm{e}}=2 \mathrm{~cm}$ ), the adiabatic section (A), and the condensation section $\left(\mathrm{C}, L_{\mathrm{c}}=3.5 \mathrm{~cm}\right)$ during the processes of startup and shutdown under $200 \mathrm{~W}$ heat input. Figure 7(b) compares the transient temperature variations of the evaporator, adiabatic, and condenser sections under the heat input 
of $200 \mathrm{~W}$ and $145 \mathrm{~W}$, respectively. The bent heat pipe is being heated for $60 \mathrm{~s}$ heat input and then continues cooling for $5 \mathrm{~min}$ with no heat input. The cold source temperature $t_{s}=80^{\circ} \mathrm{C}$. As shown in the figure, the temperature rise of the heating block is the largest. And there is a delay of thermal response for the evaporator, adiabatic, and condenser in sequence. Furthermore, as shown in Figure 7(b), when the heat input increases, the evaporator temperature shows a corresponding rise more dramatically, which demonstrates that the heat pipe can react quickly to the variation of heat input and possesses superior transient thermal performance. Moreover, the heat pipe temperature drops immediately, once the heat source is turned off. But it takes approximate 4 times as long to return to the initial temperature.

\section{Conclusions}

In this paper, an experimental system has been built to test the thermal performance of bent heat pipes. The effects of the lengths of condensation area and evaporation area and cooling temperature on the equivalent thermal conductivity and the critical heat flux of a bent copper-water heat pipe have been examined and analyzed. Moreover, the influences of heat input and working conditions on transient thermal characteristics of a bent heat pipe have also been discussed. The conclusions are as follows:

(1) As the cooling temperature increases, the critical heat flux enhances, and the temperature difference between the evaporator and condenser section remains almost unchanged when the cooling temperature changes

(2) The critical heat flux can be significantly enhanced when the length of the evaporator section is doubled regardless of changing the cooling temperature. However, the increasing cooling area shows obvious improving effect on critical heat flux when the cooling temperature is higher than $50^{\circ} \mathrm{C}$

(3) Longer heating and cooling lengths result in a higher equivalent thermal conductivity. The improvement of thermal conductivity by increasing the heating length is more evident than that by increasing length of the condensation area

(4) When the heat input is imposed to the bent copperwater heat pipe, the temperature of the evaporation area, the adiabatic area, and the condensation area will rise in sequence after the temperature of the heating block increases. A longer time is needed for the bent heat pipe to return to the initial status after the heat input is shut off when compared with the startup process

Note that for better understanding of the changes in the performance of the bent heat pipe, in the future, it is necessary to understand the heat and mass transfer characteristics by building a theoretical model considering the heat transfer and fluid flow inside the bent heat pipe. In addition, for the application of the bent heat pipe, it is also important to conduct further investigation on the effect of working conditions including the condensation temperature, bent angle, and the length of each section on the thermal performance of bent heat pipe for obtaining the optimum thermal performance of a bent heat pipe.

\section{Nomenclature}

Gr: The Grashof number (1)

$h$ : The convective heat transfer coefficient $\left(\mathrm{W} / \mathrm{m}^{2} \cdot \mathrm{K}\right)$

$H: \quad$ Performance-price coefficient $\left(\mathrm{W} /\left(\mathrm{m}^{2} \cdot \mathrm{K}\right)\right)$

$k_{\mathrm{f}}: \quad$ The equivalent thermal conductivity $(\mathrm{W} / \mathrm{m} \cdot \mathrm{K})$

$k_{\text {eff }}$ : The thermal conductivity of the fluid $(\mathrm{W} / \mathrm{m} \cdot \mathrm{K})$

$l_{c}: \quad$ The characteristic size $(\mathrm{m})$

$L_{\text {eff }}:$ The effective length $(\mathrm{m})$

$L_{\mathrm{c}}$ : $\quad$ Condenser length $(\mathrm{m})$

$L_{\mathrm{a}}: \quad$ Adiabatic section length (m)

Nu: The Nusself number (1)

Pr: The Prandtl number (1)

$Q_{\text {loss }}:$ The heat loss $(\mathrm{W})$

$Q_{\text {in }}$ : Input power $(\mathrm{W})$

$t_{\mathrm{w}}$ : The temperature of the outer surface of the insulation layer $\left({ }^{\circ} \mathrm{C}\right)$

$t_{\infty}$ : Fluid temperature $\left({ }^{\circ} \mathrm{C}\right)$

$t_{\mathrm{w}, \mathrm{e}}: \quad$ The mean temperature of the evaporator $\left({ }^{\circ} \mathrm{C}\right)$

$t_{\mathrm{w}, \mathrm{c}}$ : The mean temperature of the condenser $\left({ }^{\circ} \mathrm{C}\right)$

$T_{m}:$ The characteristic temperature $\left({ }^{\circ} \mathrm{C}\right)$.

Greek Symbols

$\Delta:$ Difference of temperature

$\alpha_{\mathrm{v}}$ : The volume expansion coefficient $\left(\mathrm{K}^{-1}\right)$.

\section{Subscripts}

a: Adiabatic section

c: Condenser

cr: Critical

e: Evaporator

eff: Effective

f: $\quad$ Fluid

in: Input

out: Outside

w,c: Wall temperature of condenser

w,e: Wall temperature of evaporator.

\section{Data Availability}

The data used to support the findings of this study are available from the corresponding author upon request.

\section{Conflicts of Interest}

The authors declare no conflict of interest. 


\section{Acknowledgments}

The authors acknowledge the financial support of this work by the National Natural Science Foundation of China (Nos. 51876184 and 51906170), Natural Science Foundation of Jiangsu Province (No. BK20180102), Natural Science Foundation of the Jiangsu Higher Education Institutions of China (No. 19KJB470008), Six Talent Peaks Project in Jiangsu Province (No. JNHB-074), China Postdoctoral Science Foundation (Nos. 2017M621835 and 2019T120469), and Jiangsu Planned Projects for Postdoctoral Research Funds (No. 1701188B).

\section{References}

[1] S. Kummerl, C. Devries, U. Chaudhry, and C. H. Lim, "Increase power density and simplify designs using 3-D sip modules," in Book Increase Power Density and Simplify Designs Using 3-D Sip Modules, International Microelectronics Assembly and Packaging Society, 2018.

[2] C. Ó. Mathúna, S. Kulkarni, Z. Pavlovic et al., "Power inside-applications and technologies for integrated power in microelectronics," in Book Power inside-Applications and Technologies for Integrated Power in Microelectronics, IEEE, 2017.

[3] Y. P. Chen, W. Gao, C. B. Zhang, and Y. J. Zhao, “Threedimensional splitting microfluidics," Lab on a Chip, vol. 16, no. 8, pp. 1332-1339, 2016.

[4] Y. Chen, C. Zhang, M. Shi, and Y. Yang, "Thermal and hydrodynamic characteristics of constructal tree-shaped minichannel heat sink," AICHE Journal, vol. 56, no. 8, pp. 2018-2029, 2010.

[5] X. Yang, Y. Yan, and D. Mullen, "Recent developments of lightweight, high performance heat pipes," Applied Thermal Engineering, vol. 33, pp. 1-14, 2012.

[6] G. E. Moore, "Progress in digital integrated electronics," in Book Progress in Digital Integrated Electronics, IEEE, 1975.

[7] L. Sun, J. Shen, Q. Hua, and K. Y. Lee, "Data-driven oxygen excess ratio control for proton exchange membrane fuel cell," Applied Energy, vol. 231, pp. 866-875, 2018.

[8] J. B. Bernstein, A. Bensoussan, and E. Bender, "Reliability prediction with Mtol," Microelectronics Reliability, vol. 68, pp.9197, 2017.

[9] C. B. Zhang, F. W. Yu, X. J. Li, and Y. P. Chen, "Gravity-capillary evaporation regimes in microgrooves," AICHE Journal, vol. 65, no. 3, pp. 1119-1125, 2019.

[10] S. Yao, Z. Ma, L. Luo, and R. Chen, "Improvement of heat pipe technique for high heat flux electronics cooling," Journal of East China Shipbuilding Institue (Natural Science Edition), vol. 17, no. 4, pp. 9-12, 2003.

[11] R. C. Chu, R. E. Simons, M. J. Ellsworth, R. R. Schmidt, and V. Cozzolino, "Review of cooling technologies for computer products," IEEE Transactions on Device and Materials Reliability, vol. 4, no. 4, pp. 568-585, 2004.

[12] S. V. Garimella, A. S. Fleischer, J. Y. Murthy et al., "Thermal challenges in next-generation electronic systems," IEEE Transactions on Components and Packaging Technologies, vol. 31, no. 4, pp. 801-815, 2008.

[13] R. L. Webb, "Next generation devices for electronic cooling with heat rejection to air," Journal of Heat Transfer, vol. 127, no. 1, pp. 2-10, 2005.
[14] J. Wang, W. Gao, H. Zhang, M. Zou, Y. Chen, and Y. Zhao, "Programmable wettability on photocontrolled graphene film," Science Advances, vol. 4, no. 9, article eaat7392, 2018.

[15] C. Y. Tsai, H. T. Chien, P. P. Ding, B. Chan, T. Y. Luh, and P. H. Chen, "Effect of structural character of gold nanoparticles in nanofluid on heat pipe thermal performance," Materials Letters, vol. 58, no. 9, pp. 1461-1465, 2004.

[16] S.-W. Kang, W.-C. Wei, S.-H. Tsai, and C.-C. Huang, "Experimental investigation of nanofluids on sintered heat pipe thermal performance," Applied Thermal Engineering, vol. 29, no. 5-6, pp. 973-979, 2009.

[17] C. Zhang, Y. Chen, R. Wu, and M. Shi, "Flow boiling in constructal tree-shaped minichannel network," International Journal of Heat and Mass Transfer, vol. 54, no. 1-3, pp. 202209, 2011.

[18] Y. P. Chen and Z. L. Deng, "Hydrodynamics of a droplet passing through a microfluidic T-junction," Journal of Fluid Mechanics, vol. 819, pp. 401-434, 2017.

[19] T. Jankowski, F. Prenger, and A. Razani, "Experimental study of a curved rotating heat pipe," Journal of Heat Transfer, vol. 130, no. 10, 2008.

[20] R. Yang and F. P. Chiang, "An experimental heat transfer study for periodically varying-curvature curved- pipe," International Journal of Heat and Mass Transfer, vol. 45, no. 15, pp. 3199-3204, 2002.

[21] B. Holley and A. Faghri, "Permeability and effective pore radius measurements for heat pipe and fuel cell applications," Applied Thermal Engineering, vol. 26, no. 4, pp. 448-462, 2006.

[22] F. D. Espinosa, T. Peters, and J. Brisson, "Effect of fabrication parameters on the thermophysical properties of sintered wicks for heat pipe applications," International Journal of Heat and Mass Transfer, vol. 55, no. 25-26, pp. 7471-7486, 2012.

[23] D. Deng, D. Liang, Y. Tang, J. Peng, X. Han, and M. Pan, "Evaluation of capillary performance of sintered porous wicks for loop heat pipe," Experimental Thermal and Fluid Science, vol. 50, pp. 1-9, 2013.

[24] C. Byon and S. J. Kim, "Capillary performance of bi-porous sintered metal wicks," International Journal of Heat and Mass Transfer, vol. 55, no. 15-16, pp. 4096-4103, 2012.

[25] G. Huang, W. Yuan, Y. Tang, B. Zhang, S. Zhang, and L. Lu, "Enhanced capillary performance in axially grooved aluminium wicks by alkaline corrosion treatment," Experimental Thermal and Fluid Science, vol. 82, pp. 212-221, 2017.

[26] S. Wu, Y. Ding, C. Zhang, and D. Xu, "Improving the performance of a thermoelectric power system using a flat-plate heat pipe," Chinese Journal of Chemical Engineering, vol. 27, no. 1, pp. 44-53, 2019.

[27] F. Yu, C. Yu, J. Cao, and Y. Chen, "Experimental analysis of the evaporation regimes of an axially grooved heat pipe at small tilt angles," International Journal of Heat and Mass Transfer, vol. 126, pp. 334-341, 2018.

[28] J. Wang, "Experimental investigation of the transient thermal performance of a bent heat pipe with grooved surface," Applied Energy, vol. 86, no. 10, pp. 2030-2037, 2009.

[29] C. Yang, C. Song, W. Shang, P. Tao, and T. Deng, "Flexible heat pipes with integrated bioinspired design," Progress in Natural Science: Materials International, vol. 25, no. 1, pp. 51-57, 2015.

[30] N. Sangpab, N. Kimura, P. Terdtoon, P. Sakulchangsatjatai, N. Kammuang-lue, and M. Murakami, "Combined effect of bending and flattening on heat transfer performance of 
cryogenic sintered-wick heat pipe," Applied Thermal Engineering, vol. 148, pp. 878-885, 2019.

[31] L.-l. Jiang, Y. Tang, and M.-q. Pan, "Effects of bending on heat transfer performance of axial micro-grooved heat pipe," Journal of Central South University of Technology, vol. 18, no. 2, pp. 580-586, 2011.

[32] X. Liu and Y. Chen, "Transient thermal performance analysis of micro heat pipes," Applied Thermal Engineering, vol. 58, no. 1-2, pp. 585-593, 2013.

[33] G. S. Wong, "Approximate equations for some acoustical and thermodynamic properties of standard air," Journal of the Acoustical Society of Japan (E), vol. 11, no. 3, pp. 145-155, 1990.

[34] J. Cooper and R. Dooley, "Revised release on the Iapws industrial formulation 1997 for the thermodynamic properties of water and steam," The International Association for the Properties of Water and Steam, vol. 1, p. 48, 2007.

[35] R. J. Moffat, "Describing the uncertainties in experimental results," Experimental Thermal and Fluid Science, vol. 1, no. 1, pp. 3-17, 1988. 